\title{
Guest Editor Introduction
}

It is indisputable the significant contribution that David Sugarbaker has made to the thoracic surgery domain, which included serving as the American Association of Thoracic Surgeons' $94^{\text {th }}$ President. Following graduation from Cornell University Medical College in New York and internship at what later became Brigham and Women's Hospital, Dr Sugarbaker was awarded a two-year National Institute of Health Research Fellowship to study benign esophageal diseases at Harvard Medical School. After completing his residency at Toronto General Hospital, he returned to Brigham and Women Hospital at the age of 35 to serve as the Chief of Thoracic Surgery. Since then, he has been instrumental in the training over 80 residents, many of whom would become established experts in their respective subspecialties, as well as establishing Brigham and Women's thoracic division to be one of the best in the world. Nicknamed 'Mr Mesothelioma', Dr Sugarbaker has devoted much of his career to improve our understanding of the pathophysiology of malignant pleural mesothelioma, and has established multimodal surgical and oncological treatment options as the gold standard.

As Director of Esophageal Surgical Services at the Baylor College of Medicine, Dr Shawn Groth has dedicated his professional life to studying and treating esophageal and thoracic cancers. In addition, after training at institutions such as University of Minnesota, Brigham and Women's Hospital, and University of Pittsburgh Medical Center, Dr Groth has built up a wealth of clinical experience and academic research that has earned him a multitude of awards. He is the recipient of numerous grants, and has published extensively on esophageal and thoracic oncology. As the Co-Director of the newly created Baylor Center for Dysphagia and Swallowing Disorders, Dr Groth is strongly committed to a multi-disciplinary team approach to managing complex esophageal conditions.

David H. Tian

(Email:david.tian@mq.edu.au)

Tristan D. Yan

(Email: tristanyan@annalscts.com)

doi: 10.21037/acs.2017.03.22

Conflicts of Interest: The authors have no conflicts of interest to declare.

View this article at: http://dx.doi.org/10.21037/acs.2017.03.22 